\title{
Design of Micro-grid Protection based on The Communication System
}

\author{
Yalin $\mathrm{Li}^{1, \text { a }}$, Qianshuang Wang ${ }^{1, \mathrm{~b}}$, Jingtao Zhao ${ }^{1, \mathrm{c}^{\star}}$, Tao Hong ${ }^{1, \mathrm{~d}}$, Tao Yang ${ }^{1, \mathrm{e}}$ \\ ${ }^{1}$ NARI Technology Development Co., Ltd., Nanjing, P R China \\ aliyalin@sgepri.sgcc.com.cn, ${ }^{b}$ wangqianshuang@sgepri.sgcc.com.cn, \\ czhaojingtao@sgepri.sgcc.com.cn, ${ }^{\mathrm{d}}$ hongtao@sgepri.sgcc.com.cn, ${ }^{\mathrm{e}}$ yangtao@sgepri.sgcc.com.cn
}

Keywords: micro-grid; micro-grid protection; DG; fault location

Abstract.This paper firstly analyzes the problems in the existing micro-grid protection. Then, the paper puts forwards a new method for micro-grid protection judgment and fault location. By using the communication system, the over-current protection device can satisfy the protection-requirements of alternative and speed. It needs no significantly increase in the cost. In current protectors, this protection method downloads a script of the export of the protection in accordance with the actual situation of the distribution network. And it has more flexibility and greater range compared with differential protection. It will adapt to the new requirements as long as changing the script of the protector when the network structure changes such as single power changing to dual power.

\section{Introduction}

With the introduction of the "Comments on the work of the State Grid Corp about the service of the distributed power supply", a large amount of distribution generation systems will connect to distributed network and generate power. It will change the structure of the distributed network from a simple loop or radiation to a complicated network with multi-powers. This kind of structure will make the relay protection fulfill a lot of new requirements.

Traditional micro-grid protection mostly adopts the non-central protective devices. However, the flexible and changeable network topology will make it so difficult to get the setting value of protective device, and the single protective device with less information will also affect the protection performance. If we use multiple devices to achieve the protection requirements of the whole transformer substation, the PT and CT cable usage will be increased, at the same time, the maintenance difficulty and the price of the device will also increase. The information we can get from the system information is limited, so it's hard to make accurate judgment on the whole system fault. As a result, it is necessary to study the new type of protective device for micro-grid.

\section{Domestic and overseas actuality of micro-grid protection}

At present, domestic and foreign scholars do the research of micro grid protection aiming to minimize the influence of DG access to the traditional distribution network protection by means of relatively perfect protection method. According to the current research, there are mainly the following three ways to achieve the above objectives: 


\section{Rapid removal of DG}

When the fault occurs, it is simple and convenient to adopt the rapid DG off-grid technology. At present, some standards such as IEEE 1547 require connected DG to get off the grid before the fault and auto reclosing occurs.

Fast removal of protection setting is relatively simple. The traditional distribution network overcurrent protection can meet the requirements. However, when the distance between the protected lines is not long and the number of segments is high, the current setting value among the adjacent segment is similar; the fault location can only be distinguished by the time setting. This situation will make the protection setting time be longer when the protective device is more close to the power supply side, the fault removal rate will be also slower. The more close the protective devices to the power supply side, the greater the current when the short circuit occurs. Then it will threaten the safe operation of the devices.

As shown in Figure 1, in order to improve the power supply reliability of the load near DG, the outer network and DG run at the same time during normal operation. When the outer net has a fault, the connection will break immediately and the load power supply will be replaced by DG, forming the off-grid operation state. In accordance with the requirements of distribution network fault, DG should be quickly off the network. And then the protective device may lead switch tripping at the power side when dealing with the fault. As a result, local load will lose standby power and cause outage. This will limits the efficiency of DG, and also cause the loss of the load, directly limiting the use of new energy.

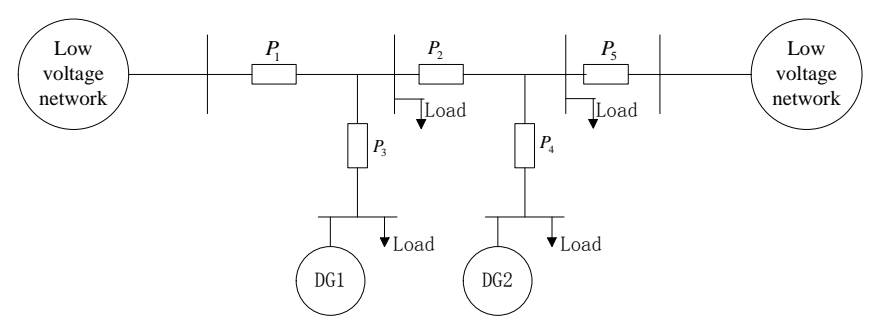

Fig.1 Distribution network diagram

\section{Micro-grid protection based on differential protection}

The differential protection is the main protection mode of electrical equipment. It has the advantages of high sensitivity, good selectivity and fast action time, and has no need to take the mutual cooperation with other segments into account. As the traditional three - stage directional overcurrent protection has been unable to meet the requirements of selectivity and fast action, the protection of the micro grid tends to adopt differential protection to solve the problem.

The Paper ${ }^{[1]}$ proposes that the differential protection is not affected by the variation of operating mode and power flow, so optical fiber differential protection can be used to solve the protection problem of distributed generation access in smart grid. But the paper also refers that optical fiber differential protection needs to access the multi-side current, which means all lines' current connecting every node should access to a differential protection device.

The paper ${ }^{[2]}$ introduces the switch station structure which is presented in Figure 2. In the figure the $3 / 2$ circuit breaker forms a switch station, where DG and the load line are combined as a component to access a string. When a component has a fault in the micro grid, the string component will be removed quickly by the differential protection. 


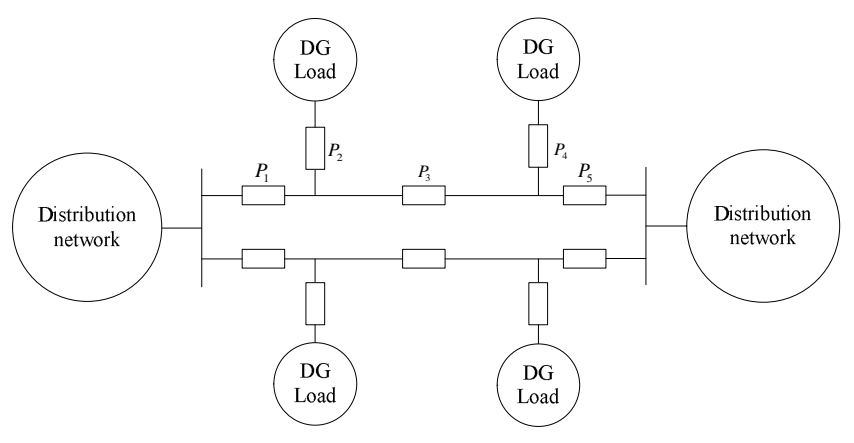

Fig.2 3/2 Circuit breaker wiring diagram

This wiring style overcomes the bad influence of the DG off-grid towards the load as is shown in the section 1.1, and this method can improve the power supply reliability of the load near the DG. But this differential protection mode also needs to access at least four line current as the calculated current in the differential protection. When accessing to multiple lines, the protection device wiring connection and the complexity of logical judgment will be much higher than the bus protection. For the micro network control station with more complexity and multi nodes, it needs several differential protection devices to achieve selective protection. What's more, in coordinate with overcurrent protection in the secondary distributed grid, the secondary bus circuit breaker must install overcurrent protective devices in the $3 / 2$ circuit breaker. This mode has a high investment cost and is of no practical significance.

\section{Micro-grid protection based on communication network}

The micro network level communication network is formed based on the communication. In order to protect micro grid, this structure carries out a comprehensive analysis and judgment using current and voltage information. The protection mode is completely dependent on communication, monitoring system or special protection management device, which needs to collect all the circuit and switch information in the micro network system. A communication interrupt or abnormal in the device will cause the whole network to lose the protection. This is not in line with the requirements of the reliability of protection. Because of the large amount of information in the faults judgment and handle, the collection time will be relatively long (general requirement of transmission speed from monitoring system to the remote changing information the information is 2 seconds). For this reason, the information processing speed and reliability of the device should be very high.

\section{Improving the performance of micro network protection by means of communication}

The micro grid protection mode is different from the traditional protection method. In the state of in-grid and off-grid, several DGs are distributed on the line. Because of the different performance of the DGs, the size of the short-circuit current is also different. Therefore, how to fast sense the faults and guarantee the selectivity, rapidity, reliability and sensitivity in the two kinds of different operating conditions of the network are the key and difficult points of the micro grid protection technology.

For micro grid protection devices, in order to judge the location of the fault point accurately and make the correct response, we should not only know the operation information of the protected object but also know the status information of other related lines and switches. Taking the rapidity of communication into account, the logic of its judgment cannot be too complicated and the 
information of other devices cannot be much, avoiding not getting the real-time signal of other related devices when faults occur.

In sum, this paper proposes a new protection mode, namely using communication system to help overcurrent protection device to achieve the protection requirements of selectivity and speed. With the development of modern communication technology, 100M / 1000M Ethernet switches are used more and more widely in micro grid automatic control and protection. In the case that the hardware cost is not increased, it can be better to meet the requirements of micro grid protection.

For the power supply network in the combination of the micro grid and the distribution network system which is shown in the figure 1, there is an overcurrent protective device at every switch. When the protective device P3 takes place over-current, we can quickly get the information of overcurrent state, switch position and the direction of the current from P1 and P2. Even if the DG itself short-circuit current is similar to the normal operating current, we still can accurately judge the fault location and make correct response. (for example: P1 has lateral fault; P2 has right side line fault; P1, P2 and P3 have the middle area fault, or P3 has micro grid internal fault location), For the protection device P3, we just know whether to act as long as we know the other two devices with a total of six remote signal information. Protective device P3 judgment logic diagram is shown in figure 3.

For the $3 / 2$ wiring power supply network in Figure 2, we only place a normal overcurrent protection device on several switches to determine the scope of the fault and isolate the fault zone.

For the micro network automation system, the number of protection device is relatively small, so all the network communication interface of the protection device needs to be connected to the same Ethernet switch. The VLAN technology, which sets the interactive transmission remote signal as the highest priority transmission level, accelerates the information transmission between protection devices. For example, in a digital substation, transmission time can be controlled to 10 milliseconds. According to the normal protection action time, the fastest export time of general instantaneous breaking protection is more than 30 milliseconds. So this method can fully meet the requirements of the speed of protection.

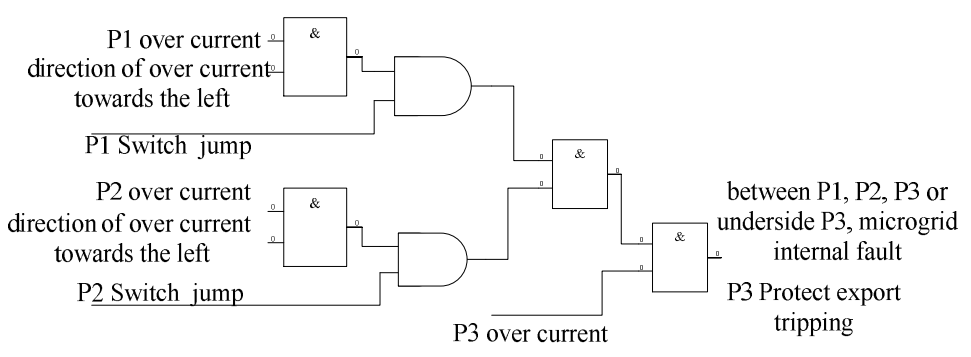

Fig.3 Protective device judgment logic diagram

For example, we suppose that each device provides 3 ports, port $\mathrm{A}$ and port $\mathrm{B}$ connects the monitor through the switch, port $\mathrm{C}$ connects $\mathrm{c}$ ports of other devices through the switch. Every device exchanges information with each other through port $\mathrm{C}$. The access to the network port $\mathrm{C}$ is achieved by using the timer interrupt query mode. Firstly, start $1 \mathrm{~ms}$ (can be set) interruption by the CPU timer, then each interrupt checks the ports to see whether there is a message, if there is, the device will immediately receive it and process and start the protection program.

For the different network card interface, the time of reading a frame message is about $10 \sim 100 \mathrm{us}$, the message size is about $1 \mathrm{~K}$, the processing and starting protection time is generally controlled at around 100us, so the information exchange between the devices can be controlled 
within the 5ms. Ignoring the switch jitter and other link delay factors, it takes about $10 \mathrm{~ms}$ to complete the whole exchange of a frame message.

For example: message sends from device 1 to device 2:

A, device 1 form a message: around 100us.

$\mathrm{B}$, write to the network card buffer: around 100us (due to the part A and B should be completed in the interrupt, therefore the maximum error is $1 \mathrm{~ms}$ which depends on the timer cycle)

$\mathrm{C}$, link transmission: around 10us, the maximum jitter of the switch will produce a maximum delay of $5 \mathrm{~ms}$

$\mathrm{D}$, device 2 reads data from the network card: around 100us

E, processing data and starting protection: around 100us

Part $\mathrm{D}$ and $\mathrm{E}$ should be completed in the interrupt, so the maximum error is $1 \mathrm{~ms}$ (depending on the timer cycle).

Total time: $2 \mathrm{~ms}+5 \mathrm{~ms}+2 \mathrm{~ms}=9 \mathrm{~ms}$. If the device uses a common network interface with the monitoring system, the transmission time will be increased, but it is entirely within the acceptable range. In current protectors, this protection method downloads a script of the export of the protection in accordance with the actual situation of the distribution network. And it has more flexibility and greater range compared with differential protection. It will adapt to the new requirements as long as changing the script of the protector when the network structure changes such as single power changing to dual power. If you encounter the entire communication system failure, each device can also continue to complete the protection function, but this time the device becomes a common three-section overcurrent protection device, the selectivity and speed of the protection is not as good as the communication system works.

\section{The promotion and influence of communication mode in the protection of micro network}

The power company has made the request to the DG access position 、 the access capacity, and using the fault current limiting device to limit the fault current and so on. When the fault occurs, the short circuit will not be big. If a large number of PV devices are installed in the distribution network, the line current may not be significantly increased when the fault occurs, so the current protection cannot work normally. What's worse, this will result in the contact overvoltage. Such a permanent fault will spread and damage more equipment, making many devices work at an unsafe operation state. However, this paper's proposed protection method can rapidly judge the fault location and isolate the fault accurately according to message of the increasing short circuit current and the changing direction of the current, which is provided by the system. Network communication is the basic communication mode in the protection device. As long as the local monitor system can provide the function of the script configuration, this method can meet all the requirements of the speed and the selectivity in the micro grid. This communication method regards every device as one of the interrelated points in the network, that is, each device is a node in the whole system and it can provide reference information for the whole network. As a result, the communication system has great importance to improve the performance of the whole network.

\section{References}

[1] Zhao Shanglin, Wu Zaijun, Hu Minqiang, Fan Yong, Thought About Protection of Distributed Generation and Microgrid [J] , Automation of Electric Power Systems , 2010,34(73-76). 
[2] Luo Jian, Ding Wanglin, Yao Zhiqiang,. Study on Relay Protection and Distributed Generation Technology in the Smart Power Grid [C]. Proceedings of international conference on uhv transmission technology. 2009.

[3] Lin Xia, Lu Yuping, Wang Lianhe. New Fault Region Location Scheme in Distribution System With DGs [J]. Transactions of China Electrotechnical Society, 2008, 11(139-145).

[4] Liu Qiang, A Probe into the Technology of Relay Protection for Smart Grid [J]. Jiangsu Electrical Enigineering, 2010,02(82-84). 